\title{
Initial Result of Neutron Emission Rate Analysis for Ion Cyclotron Range of Frequency Heated Deuterium Plasmas in LHD
}

\author{
Ryosuke SEKI ${ }^{1,2)}$, Shuji KAMIO ${ }^{1)}$, Hiroshi KASAHARA ${ }^{1)}$, Kenji SAITO ${ }^{1)}$, Tetsuo SEKI ${ }^{1)}$, \\ Kunihiro OGAWA ${ }^{1,2)}$, Mitsutaka ISOBE ${ }^{1,2)}$, Hideo NUGA ${ }^{1)}$, Hiromi TAKAHASHI ${ }^{1}$, \\ Shin $\mathrm{KUBO}^{1,3)}$, Masaki OSAKABE ${ }^{1,2)}$ and LHD Experiment Group \\ ${ }^{1)}$ National Institute for Fusion Science, National Institutes of Natural Sciences, Toki 509-5292, Japan \\ ${ }^{2}$ The Graduate University for Advanced Studies, SOKENDAI, Toki 509-5929, Japan \\ ${ }^{3)}$ Nagoya University, Nagoya 464-8601, Japan
}

(Received 25 August 2020 / Accepted 3 November 2020)

\begin{abstract}
A database of the total neutron emission rate $\left(S_{\mathrm{n}}\right)$ in the ion cyclotron range of frequency (ICRF) heated deuterium plasma in the Large Helical Device (LHD) was developed with changing the ICRF injection power and electron density. The $S_{\mathrm{n}}$ measured in the experiment was compared with the neutron emission rate calculated by the thermal reactivity of deuterium plasma to predict $S_{\mathrm{n}}$ in long-pulse discharge and to understand the heating scheme of ICRF. The ratio of $S_{\mathrm{n}}$ evaluated by calculation and that by measurement in the electron cyclotron heating $(\mathrm{ECH})$ and ICRF heating case has almost the same tendency as that of the ECH only case in which energetic deuterons are not expected. In the first campaign of deuterium ICRF plasma discharge with injection power up to $1.5 \mathrm{MW}$, neutron emission with regard to the ICRF tail deuteron was thought to be negligible. We expanded the $S_{\mathrm{n}}$ database and started building an $S_{\mathrm{n}}$ prediction code to perform a long-pulse deuterium discharge. (C) 2020 The Japan Society of Plasma Science and Nuclear Fusion Research
\end{abstract}

Keywords: neutron emission rate database, ICRF, second harmonic heating, minority proton heating, LHD

DOI: $10.1585 /$ prr.15.1202088

Steady-state high-performance plasma is one of the requirements for realizing a nuclear fusion reactor. In the large helical device (LHD), long-pulse discharge has been performed using the electron cyclotron resonance heating $(\mathrm{ECH})$ and the ion cyclotron range of frequency (ICRF) heating. Long-pulse operation with deuterium (D) gas is planned in the LHD project. The database of total neutron emission rate $\left(S_{\mathrm{n}}\right)$ in D plasma sustained by $\mathrm{ECH}$ and ICRF heating is required to perform the long-pulse D plasma discharge within the strict management of the neutron budget.

From the FY 2019 campaign, one pair of ICRF antennas was reinstalled to initiate the commissioning of the long-pulse D experiment, which is regarded as the first long-pulse D experiment performed in large-sized stellartor/helical devices. To predict Sn in the long-pulse operation, we have created the Sn database in ICRF heated D plasma in stellarator/helical devices for the first time.

In the ICRF heating experiment, neutrons are created by reactions between thermal deuterons, thermal deuteronICRF tail deuteron, and ICRF tail deuterons. Significant ICRF tail deuterons are created by second harmonic deuteron heating, whereas almost no ICRF tail deuterons are expected with proton minority heating. Therefore, the neutron emission from thermal deuterons-ICRF tail deuterons will appear when the second harmonics deuteron

author'se-mail: seki.ryohsuke@nifs.ac.jp heating is sufficient. In this study, we calculated a neutron emission rate using the thermal D-D reactivity on the basis of the ion temperature measurement. By comparing the calculated and measured neutron emission rates, the neutron emission rate owing to second harmonics deuteron heating is investigated, and we will create the Sn prediction code to perform a long-pulse deuterium discharge.

Figure 1 shows time evolution of ECH and ICRF injection powers, central electron temperature $\left(T_{\mathrm{e} 0}\right)$, line-

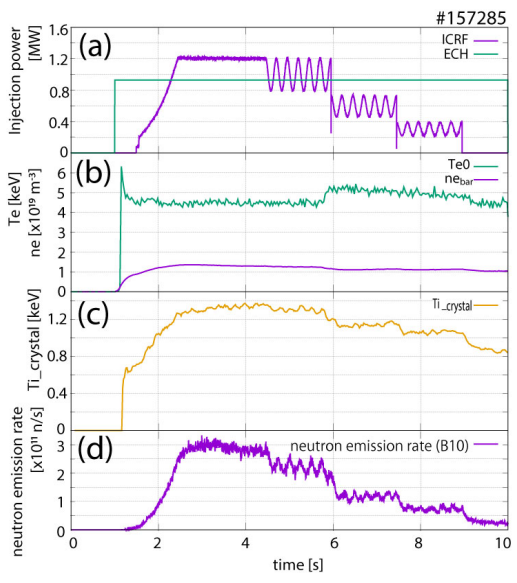

Fig. 1 Typical time evolution of ECH and ICRF heated deuterium discharge with hydrogen gas puffing. 


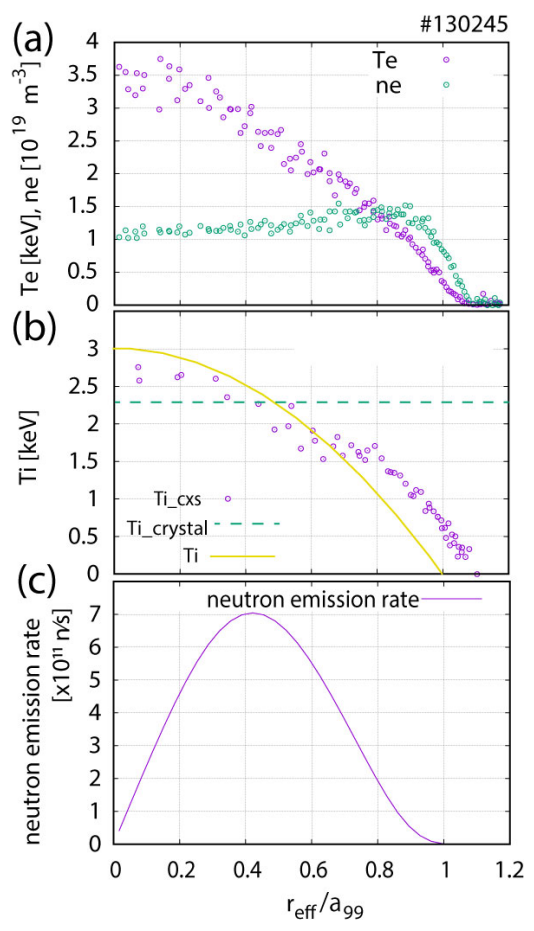

Fig. 2 Radial profiles of the (a) electron temperature and density, (b) ion temperature, and (c) neutron emission rate.

averaged electron density $\left(n_{\mathrm{e} \_ \text {bar }}\right)$, ion temperature evaluated by the Doppler width of the Ar XVII line ( $\left.T_{\mathrm{i} \_c r y s t a l}\right)$ [1], and $S_{\mathrm{n}}$ measured by the ${ }^{10} \mathrm{~B}$ counter of the neutron flux monitor $[2,3]$ in $\mathrm{D}$ plasmas. Here, the hydrogen concentration $\mathrm{H} /(\mathrm{H}+\mathrm{D})$ measured by $\mathrm{H} \alpha$ and $\mathrm{D} \alpha$ spectroscopic measurement was less than $10 \%$. The ICRF power absorption efficiency evaluated by power modulation is approximately $75 \%$. Figure 1 shows that $S_{\mathrm{n}}$ changes according to the ICRF injection power, which is expected.

Neutron emission calculation based on the thermal fusion reaction is performed to identify the neutron emission rate due to the second harmonics deuteron heating. Figure 2 (a) shows the radial profiles of electron temperature and electron density measured in a previously performed He plasma experiment sustained by ECH and ICRF minority heating, where the ion temperature was measured by charge exchange spectroscopy. In this experiment, ICRF and $\mathrm{ECH}$ powers are 4 and $1 \mathrm{MW}$, respectively. The minority ratio is $30 \%$. The ion temperature profile shown in Fig. $2(\mathrm{~b})$ is given as $T_{\mathrm{i}}=(4 / 3) T_{\mathrm{i} \_ \text {crystal }}\left(1-\left(r_{\text {eff }} / a_{99}\right)\right)^{2}$ based on the charge exchange spectroscopy $\left(T_{\mathrm{i}_{-} \mathrm{CXS}}\right)$ measurement. Here, the central ion temperature is assumed to be $(4 / 3) \times T_{\text {i_crystal }_{-}}$based on the comparison of $T_{\text {i_cXs }}$ at plasma center and $T_{\mathrm{i} \text { ccystal }}$. Figure 2 (c) shows the neutron emission rate evaluated from the ion temperature profile and deuteron density profile, which is assumed to be the same as the electron density profile. In this calculation, the electron temperature profile is not used because the neutron emission rate is independent of the electron temperature.

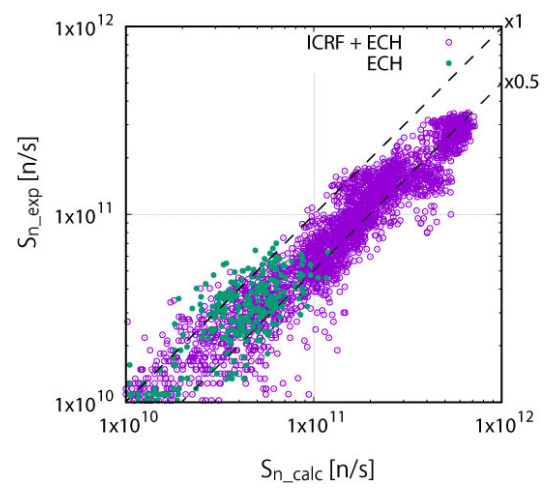

Fig. 3 Comparison of the neutron emission rate calculated using the thermal D-D reactivity and measured neutron emission rate.

In this calculation, we only integrate neutron emission up to $r_{\text {eff }}=a_{99}$, where $a_{99}$ denotes the effective minor radius in which $99 \%$ plasma stored energy is existed, because the neutron emission at $r_{\mathrm{eff}}>a_{99}$ is much smaller than that at the neutron emission peak because of the low-ion temperature. The calculated neutron emission rate from the thermal reaction $\left(S_{\mathrm{n} \_ \text {calc }}\right)$ was evaluated by

$$
S_{\mathrm{n} \_ \text {calc }}=\frac{1}{2}\left(\frac{n_{\mathrm{D}}}{n_{\mathrm{e}}}\right)^{2} \int_{V_{\mathrm{p}}} d V\left(n_{\mathrm{e}}{ }^{2}\left\langle\sigma_{\mathrm{DD}} v\right\rangle\right),
$$

where $\left\langle\sigma_{D D} v\right\rangle$ is the thermal D-D reactivity described in Ref. [4].

Figure 3 shows the comparison between the total neutron emission rate measured in the experiment $\left(S_{\mathrm{n} \_ \text {exp }}\right)$ and $S_{\mathrm{n} \_ \text {calc }}$ in D plasma sustained by ECH and ICRF heating. Here, the ICRF injection power range is from 0.2 to 1.5 MW and the electron density range is from $0.5 \times 10^{19}$ to $1.4 \times 10^{19} \mathrm{~m}^{-3}$. The deuteron density is evaluated by spectroscopy. $S_{\text {n_calc }}$ was approximately two times higher than $S_{\text {n_exp }}$. This overestimation is seen because impurity ions, such as carbon, were not considered in this calculation. The ratio of $S_{\mathrm{n} \_ \text {calc }}$ to $S_{\mathrm{n} \_ \text {exp }}$ in the D plasma sustained by ECH and ICRF heating is almost the same as that sustained by only ECH. This result implies that neutron emission from thermal-ICRF tail deuterons was not visible. In the first ICRF D campaign, the ICRF wave was thought to be mainly absorbed through minority proton heating in $\mathrm{D}$ plasma.

We will further expand $S_{\mathrm{n}}$ database in LHD D plasma sustained by higher power ICRF heating for the precise prediction of $S_{\mathrm{n}}$ in long-pulse discharge and investigation of the heating scheme of ICRF.

[1] M. Goto et al., Fusion Sci. Technol. 58, 394 (2010).

[2] M. Isobe et al., IEEE Trans. Plasma Sci. 46, 2050 (2018).

[3] K. Ogawa et al., Nucl. Fusion 59, 076017 (2019).

[4] H.S. Bosch and G.M. Hale, Nucl. Fusion 32, 611 (1992). 